\title{
NONLINEAR MAGNETIC LEVITATION OF AUTOMOTIVE ENGINE VALVES
}

\author{
K. Peterson, J.W. Grizzle, and A.G. Stefanopoulou ${ }^{*, 1}$ \\ * University of Michigan, Ann Arbor
}

\begin{abstract}
Position regulation of a magnetic levitation device is achieved through a control Lyapunov function (CLF) feedback design. The CLF is based on LQR optimal control to enhance performance. Sontag's universal stabilizing feedback is used to enhance the region of attraction. The effects of magnetic saturation are included in the model, and accounted for in the controller. While the control methodology presented here is applicable to generic magnetic levitation, the controller is designed for and implemented on an electromagnetic valve actuator for use in automotive engines.
\end{abstract}

Keywords: Electromagnetic Devices, Nonlinear Control, Automobiles

\section{INTRODUCTION}

Electromagnetic levitation is a classic control problem for which numerous solutions have been proposed. Many of the proposed solutions have focused on the use of feedback linearization (Lindlau and Knospe, 2002; Munaro et al., 2002; Mao and Li, 1999; Charara et al., 1996; Fabien, 1996; Smith and Weldon, 1995) due to the nonlinear characteristics of the magnetic and electric subsystems. Unfortunately, feedback linearization requires a very accurate model which may be unrealistic near the electromagnet due to magnetic saturation and eddy current effects, thereby limiting the range of motion achievable in the closed-loop system. Sliding mode (Rundell et al., 1996; Charara et al., 1996; Cho et al., 1993) and $H_{\infty}$ (Yi et al., 1995; Shibukawa et al., 1991) control have been used to account for the changing local dynamics and to provide robustness against unmodeled nonlinearities present in the system. Linearization and switching can be avoided through the application of nonlinear control designs based on backstepping (Green and Craig, 1998), passivity (Velasco-Villa et al., 2001), or saturation functions (Gentili and Marconi, 2003).

The control design investigated here is based on control Lyapunov functions (CLF) and Sontag's universal

\footnotetext{
${ }^{1}$ Support is provided by NSF and Ford Motor Company
}

stabilizing feedback (Sontag, 1989). The control Lyapunov function is selected on the basis of a solution to an algebraic Riccati equation to allow us to "tune" the controller for performance. Neither current control nor a static relationship between current, voltage, and the magnetic force is assumed, as done by VelascoVilla (Velasco-Villa et al., 2001) and Green (Green and Craig, 1998). Instead, the dynamics of the current/flux are compensated for through the use of a full-state feedback/observer structure rather than the inner loop/outer loop control structure proposed by Gentili (Gentili and Marconi, 2003). In addition, the effects of magnetic saturation are included to improve performance and robustness near the electromagnets. Implementation is achieved using position and current sensors, a nonlinear observer to estimate velocity, a novel method to estimate the magnetic flux, and voltage control. To demonstrate the effectiveness of the controller, it is experimentally evaluated on an electromagnetic valve actuator, designed for use in the actuation of automotive engine valves.

\section{ELECTROMAGNETIC VALVE ACTUATOR}

The electromagnetic valve actuator (EVA), shown in the experimental setup of Fig. 1, has received significant attention recently due to its potential to improve the performance of the standard internal combustion 
engine. Currently, most automotive engine valves are actuated via a camshaft. While this has proven to be a robust solution for many years, it represents a trade off between stability, robustness, and performance as it imposes a mechanical coupling between the valve timing and engine speed. By replacing the camshaft with the EVA, which uses electromagnets to actuate the engine valves, the valve timing can be decoupled from the engine speed allowing for improvements in fuel economy, torque, and emissions (Levin and Schechter, 1996). While numerous actuators have been proposed to achieve variable valve timing, the EVA has been singled out for its potential to achieve continuously variable timing and lift. With the exception of our previous work (Peterson and Stefanopoulou, 2003), research on the control of the EVA has primarily focused on the elimination of large impacts that occur between the armature and lower and upper magnetic coils. In this paper we expand on our previous work (Peterson and Stefanopoulou, 2003), which only briefly touched on the subject of magnetic levitation.

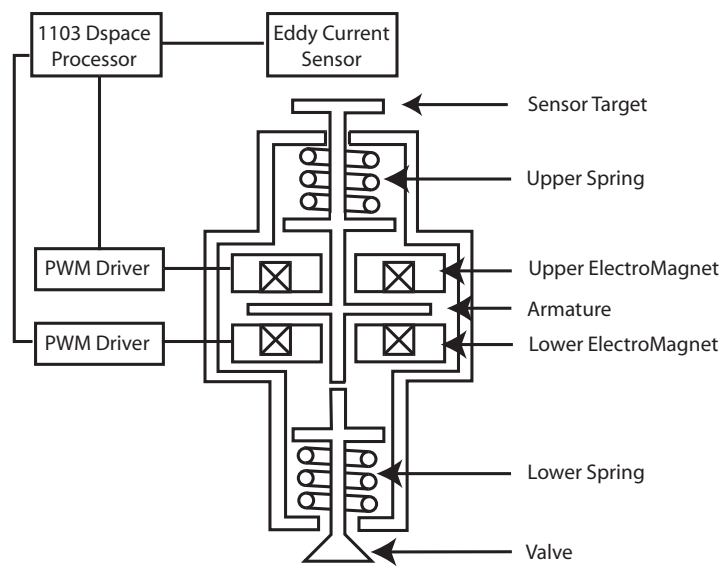

Fig. 1. Electromagnetic valve actuator and experimental setup.

The electromagnetic valve actuator governs the valve motion in the following manner. The armature is initially held against the upper magnetic coil, causing the valve to seal off the engine cylinder. In this position, the upper spring is more compressed than the lower spring, thereby creating a force imbalance as the upper and lower springs are identical. When the valve open command is issued, the current in the upper magnetic coil is reduced to zero and the potential energy stored in the upper spring drives the armature downward, which in turn causes the valve to open. Depending on the command to the actuator, two outcomes are possible. In the case of full valve opening, the lower magnetic coil is used to bring the armature in contact with the lower electromagnet, and held there. From the view point of stability, this is the more trivial case as the armature can be held against the lower magnetic coil by applying a sufficiently large constant voltage command. In the case of partial valve lift, the electromagnets are used to hover the armature. When the valve closed command is issued, the upper magnetic coil and any potential energy stored in the lower spring are used to return the armature/valve to the closed position.

Hovering the armature is slightly different than the classic control problem of magnetic levitation. Due to the presence of the springs, the equilibrium point of the armature is equidistant from either electromagnet when both electromagnets are de-energized. Hence, the upper magnetic coil is used to hover the armature above the mid-position and the lower magnetic coil is used to hover the armature below the mid-position. As shown by Tai (Tai and Tsao, 2002), any equilibrium position that is greater than $1 / 3$ the full lift away from either electromagnet is open-loop stable. However, the presence of the springs does not necessarily make the control problem appreciably easier. As the springs are extremely stiff, the bandwidth of the mechanical subsystem is on the order of the bandwidth of the electrical subsystem. Therefore, it is not practical to assume current control as will be shown in Sec. 4. In addition, the region of attraction of the open-loop stable equilibria are small. It was found experimentally that the armature had to start at rest equidistant from both electromagnets to achieve stable open-loop hovering for equilibrium positions greater than $1 / 3$ the full lift away from either electromagnet. If the armature starts at rest against the upper magnetic coil, application of the open-loop equilibrium voltage results in the armature being brought into contact with the activated electromagnet. As the latter initial condition is the one experienced during operation, it is necessary to design a closed-loop controller to enlarge the region of attraction so that hovering is achieved for both openloop stable and unstable equilibrium positions.

\section{MODELING THE ELECTROMAGNETIC VALVE ACTUATOR}

As derived by Wang (Wang, 2001) the dynamics of the EVA are given by

$$
\begin{aligned}
\frac{d z}{d t} & =v \cdot 10^{3} \\
\frac{d v}{d t} & =\frac{1}{m}\left(F_{m a g}^{u}-F_{m a g}^{l}+k_{s}(l-z)-b v\right) \\
\frac{d \lambda_{l}}{d t} & =\left(V_{l}-r i_{l}\right) \cdot 10^{3} \\
\frac{d \lambda_{u}}{d t} & =\left(V_{u}-r i_{u}\right) \cdot 10^{3}
\end{aligned}
$$

where $z(\mathrm{~mm})$ is the distance from the lower coil, $v(\mathrm{~m} / \mathrm{s})$ is the velocity of the armature, $\lambda_{l}(\mathrm{mVs})$ is the magnetic flux in the lower coil, $\lambda_{u}(\mathrm{mVs})$ is the magnetic flux in the upper coil, $m(\mathrm{~kg})$ is the combined mass of the armature and valve, $F_{m a g}^{l}(\mathrm{~N})$ is the magnetic force generated by the lower coil, $F_{m a g}^{u}(\mathrm{~N})$ is the magnetic force generated by the upper coil, $k_{s}$ 
$(\mathrm{N} / \mathrm{mm})$ is the spring constant, $l(\mathrm{~mm})$ is half the total armature travel, $b(\mathrm{~kg} / \mathrm{s})$ is the damping coefficient, $V_{l}$ $(\mathrm{V})$ is the voltage applied to the lower coil, $V_{u}(\mathrm{~V})$ is the voltage applied to the upper coil, $r(\Omega)$ is the combined resistance of the wiring and magnetic coil, $i_{l}$ (A) is the current in the lower coil, and $i_{u}(\mathrm{~A})$ is the current in the upper coil.

The current and the magnetic flux are related through

$$
N i=\frac{\lambda}{N}\left(\Re_{\text {core }}+\Re_{\text {arm }}+\Re_{\text {gap }}\right)
$$

where $N$ is the number of turns in the magnetic coil and $\Re_{\text {core }}, \Re_{\text {arm }}, \Re_{\text {gap }}$ are the reluctances of the magnetic core, armature, and air gap as given by Wang (Wang, 2001).

The reluctance of the armature and core are approximately constant and the reluctance of the air gap is proportional to the distance between the electromagnet and armature. Magnetic saturation is included in the model by multiplying $\Re_{\text {core }}$ by

$$
\frac{\lambda_{\max }}{\lambda_{\max }-\lambda}
$$

as suggested by Peek (Peek and Wagar, 1951) where $\lambda_{\max }$ is the maximum magnetic flux. Therefore, the relationship between current and flux is

$$
i_{l}=\frac{\lambda_{l}}{k_{a}}\left(k_{b} \frac{\lambda_{\max }}{\lambda_{\max }-\lambda_{l}}+k_{c}+z\right) .
$$

The flux and current in the upper coil are related by a similar relationship where the position is shifted by $2 l$.

Using this relationship, the magnetic force is determined via an energy balance. The work done by the magnetic force must equal the change in electrical energy. Thus

$$
\int F_{m a g} d z=\int i d \lambda \Rightarrow F_{m a g}=\frac{\lambda^{2}}{2 k_{a}}
$$

As explained in Sec. 2, only one electromagnet is used to hover the armature. Therefore, without loss of generality we assume that the lower coil is used and neglect the dynamics of the upper coil. The system dynamics are then written as

$$
\begin{aligned}
\frac{d z}{d t} & =v \cdot 10^{3} \\
\frac{d v}{d t} & =\frac{1}{m}\left(-\frac{\lambda_{l}^{2}}{2 k_{a}}+k_{s}(l-z)-b v\right) \\
\frac{d \lambda_{l}}{d t} & =\left(V_{l}-\frac{r \lambda_{l}}{k_{a}}\left(k_{b} \frac{\lambda_{\max }}{\lambda_{\max }-\lambda_{l}}+k_{c}+z\right)\right) \cdot 10^{3}
\end{aligned}
$$

To simplify the controller design, new inputs and coordinates are introduced per $x_{1}=z-z_{e q}, x_{2}=v-$ $v_{e q}, x_{3}=\lambda_{l}-\lambda_{e q}$, and $u=V_{l}-V_{e q}$ where $z_{e q}, v_{e q}$, $\lambda_{e q}$, and $V_{e q}$ are the equilibrium position, velocity, flux, and voltage respectively.

The equilibrium point is now located at the origin, and the system dynamics are represented in the form $\frac{d x}{d t}=f(x)+g(x) u$ as

$$
\begin{aligned}
& \frac{d x}{d t}= \underbrace{\left[\begin{array}{c}
x_{2} \cdot 10^{3} \\
\frac{1}{m}\left(-\frac{x_{3}^{2}}{2 k_{a}}-k_{s} x_{1}-b x_{2}\right)-\frac{x_{3} \lambda_{e q}}{k_{a} m} \\
\left(V_{e q}-\frac{r\left(x_{3}+\lambda_{e q}\right)}{k_{a}} \Psi\left(x_{1}, x_{3}\right)\right) \cdot 10^{3}
\end{array}\right]}_{f(x)}+ \\
& \underbrace{\left[\begin{array}{c}
0 \\
0 \\
10^{3}
\end{array}\right]}_{g(x)} u
\end{aligned}
$$

where

$$
\Psi\left(x_{1}, x_{3}\right)=\frac{k_{b} \lambda_{\max }}{\lambda_{\max }-x_{3}-\lambda_{e q}}+k_{c}+x_{1}+z_{e q} .
$$

\section{CONTROLLER DESIGN}

The proposed controller consists of two components, a stabilizing controller to achieve hovering and a damping controller that retards the initial motion of the armature during release. The stabilizing controller is based on Sontag's universal stabilizing feedback (Sontag, 1989). The control Lyapunov function (CLF) is chosen based on LQR control for performance. Since the electromagnet can only apply attractive forces, the upper coil is used to slow the release of the armature to reduce overshoot near the lower coil.

\subsection{Stabilizing Controller}

A frequent assumption in the control of electromagnetic actuators is that the dynamics of the current/flux are significantly faster than the dynamics of the mechanical components of the system. This assumption reduces the complexity of the control problem as most of the nonlinearities are present in the dynamics of current/flux. Unfortunately, this assumption is not valid for our system. The bandwidth of position is approximately equal to the bandwidth of current/flux due to the presence of stiff springs.

As the flux dynamics are non-negligible, we have used Sontag's feedback (Sontag, 1989) to stabilize the three state system. Given a CLF, V, the feedback

$$
u=\left\{\begin{array}{cc}
-\frac{L_{f} V+\sqrt{L_{f} V^{2}+L_{g} V^{4}}}{L_{g} V} & \text { for } L_{g} V \neq 0 \\
0 & \text { for } L_{g} V=0
\end{array}\right.
$$


proposed by Sontag, will render the origin asymptotically stable, where $L_{f} V$ and $L_{g} V$ are the Lie derivatives of the CLF, defined as $L_{f} V=\frac{\partial V}{\partial x} f(x)$ and $L_{g} V=\frac{\partial V}{\partial x} g(x)$.

In our previous work (Peterson and Stefanopoulou, 2003), we proposed a CLF for which Sontag's feedback rendered the electromagnetic valve actuator asymptotically stable. Unfortunately, there is no intuitive method to modify our previous CLF to influence the performance of the system. For this reason, a better candidate CLF is sought.

Let us instead select the CLF to be

$$
V=x^{T} P x
$$

where the matrix $P$ satisfies the algebraic Riccati equation

$$
P A+A^{T} P+Q-P B B^{T} P=0
$$

for

$$
A=\left.\frac{\partial f(x)}{\partial x}\right|_{x=0} \text { and } B=\left.g(x)\right|_{x=0}
$$

as suggested by Sepulchre (Sepulchre et al., 1997). The motivation is that we can select the matrix $P$ by manipulating the cost function

$$
J=\int_{0}^{\infty}\left(x^{T} Q x+u^{2}\right) d t
$$

of an LQR optimal control problem subject to the dynamics

$$
\frac{d x}{d t}=A x+B u
$$

We can therefore hopefully influence the performance of the system in a direct way. Indeed, it will be shown in Sec. 5 that selecting the Lyapunov function in this manner allows us to easily adjust the apparent damping of the closed-loop system. Note that even though we are using a quadratic CLF, the Lie derivatives are constructed using the nonlinear system dynamics.

\subsection{Damping the Release}

When released from rest against the upper coil, the armature swings to within $0.5 \mathrm{~mm}$ of the lower coil if the upper coil is not used to slow the motion of the armature. As the electromagnets are only capable of applying attractive forces, the controller outlined in Sec. 4.1 is unable to reduce any resulting overshoot when hovering below the mid-position. The obvious solution would be to select a multi-input CLF so the feedback could utilize the upper coil to reduce the overshoot. Unfortunately, it is not practical to select a multi-input CLF using the method explained in Sec. 4.1 due to the dynamics of our system. The effects of the second coil on the motion of the armature are lost when the system is linearized as the magnetic force is proportional to the square of the flux and the equilibrium flux is zero. Therefore the upper coil will not be utilized to minimize the cost function given in Eqn. (7).

Instead, to overcome the overshoot, the upper coil is used in the following manner when hovering below the mid-position. First, the flux in the upper coil is reduced to zero to release the armature. Afterward, the upper coil is used as a virtual damper once the armature has moved $0.1 \mathrm{~mm}$ away from it. The is achieved by setting the desired magnetic force generated by the upper coil proportional to the velocity of the armature

$$
F_{m a g}^{u}=-\alpha v
$$

and thus the desired flux in the upper coil equal to

$$
\lambda_{u}=\sqrt{-2 k_{a} \alpha v}
$$

where the value of $\alpha$ is given in Table 1 . The duty cycle command to the PWM driver of the upper coil is set to either full on, if the measured flux is less than the desired flux, or full reverse, if the measured flux is greater than the desired flux. To avoid taking the square root of a negative number, the upper coil is deactivated, and remains de-activated, when the velocity of the armature returns to zero. As the armature has just started to move when the damping controller is turned on, the velocity is approximately zero and thus the desired flux in the upper coil is approximately zero. The damping controller then retards the release of the armature until the velocity is once again zero at which point it shuts off permanently. The desired flux in the upper coil is also zero at this point as it is proportional to the velocity. During this entire time the hovering controller described in Sec. 4.1 is active. It was decided not to include the effects of the damping controller in the linearized model used to choose the CLF as the damping controller will permanently turn the upper coil off when the armature velocity reaches zero.

\subsection{Nonlinear Observer}

As the actuator is intended for use in an automotive engine it is impractical to measure every state. The available measurements are the position of the armature and the current in each coil. Current is chosen over flux as current sensors are standard in most power electronic devices and the compact design of the actuator prevents the mounting of hall effect sensors.

First, the magnetic flux is determined using a map of the magnetic force as a function of current and 
position developed by Wang (Wang, 2001), denoted as $F_{m a g}(i, z)$. Given the position and current, the magnetic flux is determined by

$$
\lambda=\sqrt{2 k_{a} F_{m a g}(i, z)}
$$

based on Eqn (4). Second, the armature velocity is estimated by the nonlinear observer

$$
\begin{aligned}
& \frac{d \widehat{z}}{d t}=\widehat{v}+\Gamma_{1}(z, \widehat{z}) \\
& \frac{d \widehat{v}}{d t}=\frac{1}{m}\left(k_{s}(l-\widehat{z})-b \widehat{v}\right)+\Gamma_{2}\left(i_{l}, i_{u}, z, \widehat{z}\right),
\end{aligned}
$$

where

$$
\begin{aligned}
\Gamma_{1}(z, \widehat{z})= & g_{1}(z-\widehat{z}) \\
\Gamma_{2}\left(i_{l}, i_{u}, z, \widehat{z}\right)= & F_{m a g}^{u}\left(i_{u}, z\right)-F_{m a g}^{l}\left(i_{l}, z\right) \\
& +g_{2}(z-\widehat{z}) .
\end{aligned}
$$

Computing the error dynamics, $e=\left[\begin{array}{l}z-\widehat{z} \\ v-\widehat{v}\end{array}\right]$, results in

$$
\begin{aligned}
\frac{d}{d t}\left[\begin{array}{l}
e_{1} \\
e_{2}
\end{array}\right]= & \underbrace{\left[\begin{array}{cc}
0 & 10^{3} \\
-k_{s} / m & -b / m
\end{array}\right]}_{A_{r}}\left[\begin{array}{l}
e_{1} \\
e_{2}
\end{array}\right] \\
& +\left[\begin{array}{l}
g_{1} \\
g_{2}
\end{array}\right] \underbrace{\left[\begin{array}{ll}
1 & 0
\end{array}\right]}_{C_{r}}\left[\begin{array}{l}
e_{1} \\
e_{2}
\end{array}\right]
\end{aligned}
$$

where the pair $\left(A_{r}, C_{r}\right)$ is observable. Values for the output injection gains $g_{1}$ and $g_{2}$ are given in Table. 1.

In its final form, the controller is implemented using the measured position, the estimated velocity from the observer, and the approximated flux using Eqn. (11) based on the measured position and current.

\section{EXPERIMENTAL RESULTS}

The effectiveness of the controller outlined in Sec. 4 is determined through implementation on the experimental setup shown in Fig. 1. The control algorithm is encoded on the dSpace 1103 processor. The processor determines the position of the armature from the eddy current sensor, that detects changes in a selfgenerated magnetic field caused by the motion of the sensor target. The current in each coil is measured by built-in sensors in the PWM drivers. Based on these signals, the processor specifies the duty cycle to each PWM driver to control the motion of the armature. We assume that the PWM drivers are sufficiently fast that the applied voltage is equivalent to the commanded duty cycle multiplied by the supply voltage of $180 \mathrm{~V}$.

The performance of the controller is presented in Figs. 2 and 3, which show the armature hovering at several different equilibrium positions. The upper magnetic coil is located at $z=8 \mathrm{~mm}$ and the lower magnetic coil is located at $z=0 \mathrm{~mm}$. The signal shown is the unfiltered position based on the eddy current sensor mounted on the rear of the actuator. The $Q$ matrices used in conjunction with Eqn. (7) to select the CLFs for each equilibrium point are given in Table. 2. Recall from Sec. 2 that all equilibrium points less $1 / 3$ the total lift (approximately $2.6 \mathrm{~mm}$ ) away from either electromagnet are open-loop unstable. As shown in Fig. 2, we are able to hover the armature approximately $1 \mathrm{~mm}$ away from either electromagnet. In a few of the set points, there is a small amount of steady state tracking error. We hope to eliminate this in future control designs with the addition of an integrator. Convergence of the velocity estimation is shown in Fig. 4.

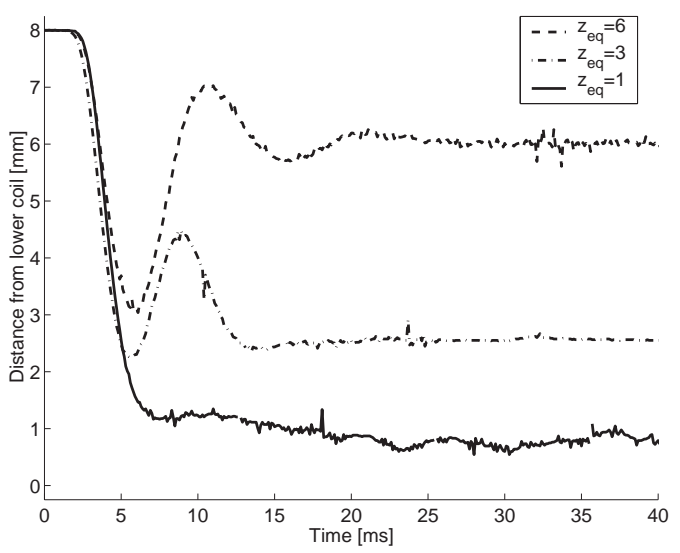

Fig. 2. Armature hovering at $1 \mathrm{~mm}, 3 \mathrm{~mm}$, and $6 \mathrm{~mm}$ away from the lower coil.

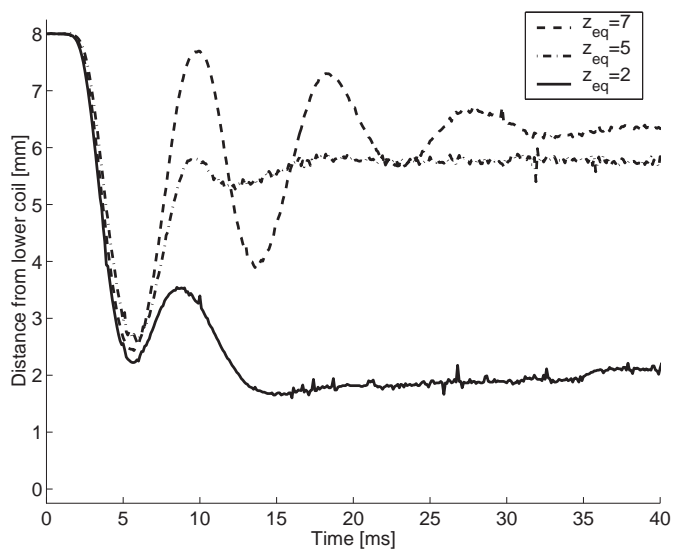

Fig. 3. Armature hovering at $2 \mathrm{~mm}, 5 \mathrm{~mm}$, and $7 \mathrm{~mm}$ away from the lower coil.

The effect of tuning the matrix $Q$ from Eqn. (7) is seen in Fig. 5. The hovering point has been set to the midposition and the matrix $Q$ for $z_{e q}=4$ is used; see Table 2. The $Q(2,2)$ element is varied according to the value given on the $y$-axis of each subplot. Manipulating the penalty on the velocity state significantly increases the effective closed-loop damping. It should be noted that the higher oscillations are not caused by the controller, but rather the closed-loop response 


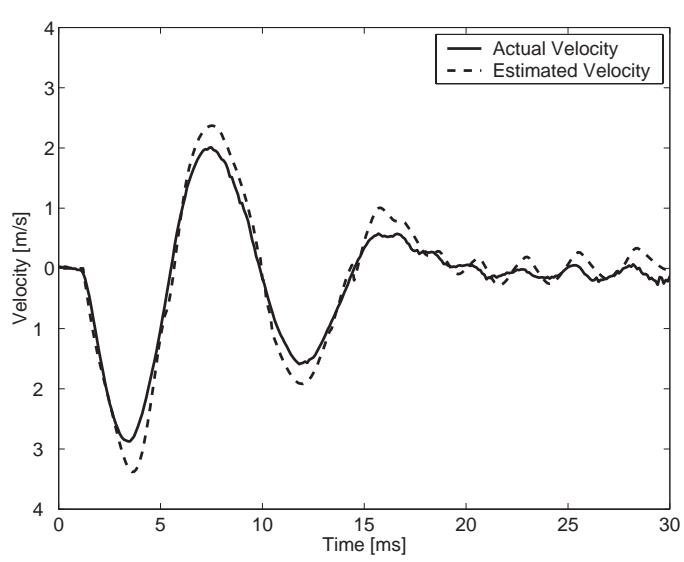

Fig. 4. Comparison of the actual vs. estimated velocity.

approaches the free response of the system as $Q(2,2)$ is reduced.
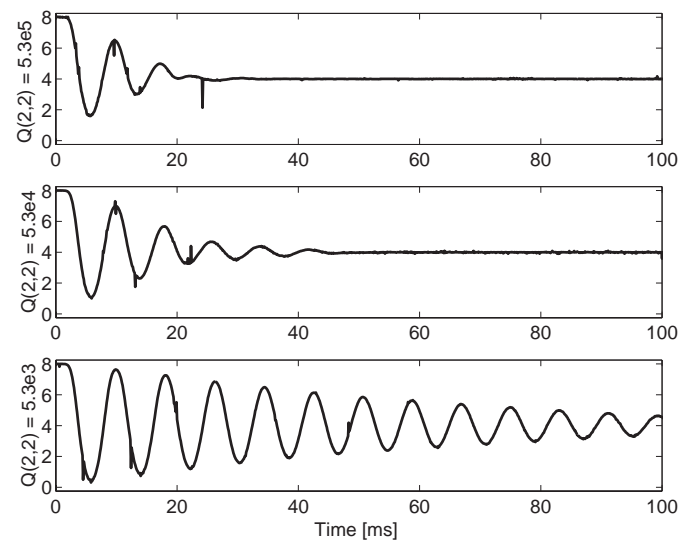

Fig. 5. Effects of tuning the matrix Q.

Hovering closer than $1 \mathrm{~mm}$ away from the electromagnet is problematic due to noise in the current sensor. As seen in the top half of Fig. 6, the controller occasionally fails to stabilize the armature at $1 \mathrm{~mm}$ due to noise in the flux measurement. Ignoring the effects of saturation, the flux estimation based on Eqn. (11) is

$$
\lambda=\frac{k_{a} i}{k_{b}+k_{c}+z}+\frac{k_{a} \Delta i}{k_{b}+k_{c}+z} .
$$

where $\Delta i$ is the noise in the current measurement. Therefore near the magnetic coil (i.e. $z \rightarrow 0$ ) any noise in the current measurement will be magnified in the approximation of flux since $k_{b}$ and $k_{c}$ are much less than 1, as seen in the top half of Fig. 6. Away from the magnetic coil (i.e. $z>1$ ) the effect of current measurement noise is not as problematic as the denominator of Eqn. (12) is greater than 1, as seen in the bottom half of Fig. 6. There is little that can be done to filter the flux due to its low frequency content; see Fig. 7. As expected there is a small peak at $60 \mathrm{~Hz}$, but most of the noise is below $40 \mathrm{~Hz}$.

As stated in Sec. 2, Tai (Tai and Tsao, 2002) has previously shown that the EVA is unstable for all equilibrium positions less than $2 l / 3 \mathrm{~mm}$ away from either

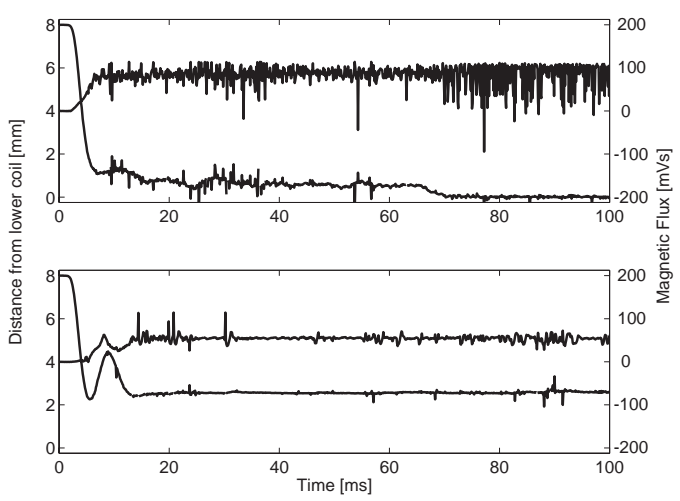

Fig. 6. Difficulties of hovering at or below $1 \mathrm{~mm}$ away from the electromagnet.
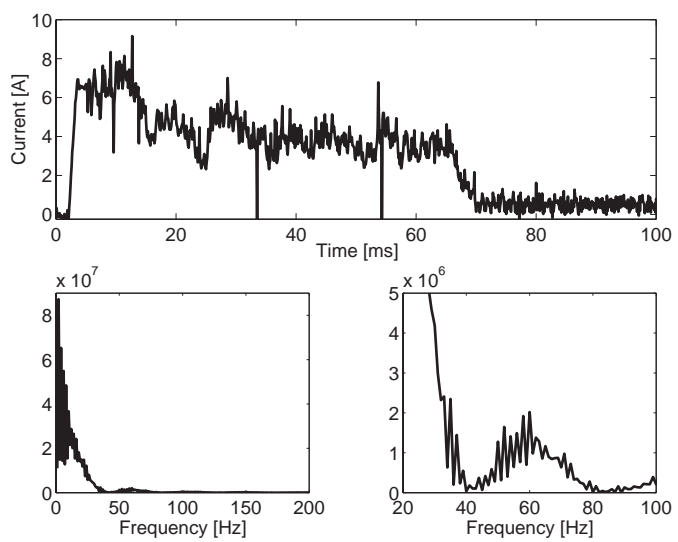

Fig. 7. Current and its FFT.

electromagnet. Based on how we have defined position, the equilibrium positions in the range $2.6 \mathrm{~mm}<$ $z<5.3 \mathrm{~mm}$ are open-loop stable. Nevertheless, it is difficult to achieve hovering in this range using open-loop control as the equilibrium positions have a small region of attraction. If the armature starts at rest against the upper magnetic coil (which is the normal mode of operation), application of the open-loop voltage for $\mathrm{z}=2.6 \mathrm{~mm}$ causes the armature to collide and remain in contact with the lower coil; see Fig. 8. To achieve stable open-loop hovering at $\mathrm{z}=2.6 \mathrm{~mm}$ the armature must be started at rest equidistant from either magnetic coil ( $z=4 \mathrm{~mm}$ ) before application of the open loop voltage; see Fig. 8. As shown in Figs. 2 and 3, the closed-loop controller has extended the region of attraction of the open-loop stable equilibrium positions. The system is now capable of achieving stable hovering in this range when the armature begins at rest against the upper magnetic coil, which is the condition experienced in operation.

\section{CONCLUSION}

Stable hovering is achieved for a wide range of lift conditions for an electromagnetic valve actuator using Sontag's feedback. Experimentally, we found it possible to influence the performance of the system by selecting the control Lyapunov function based on 


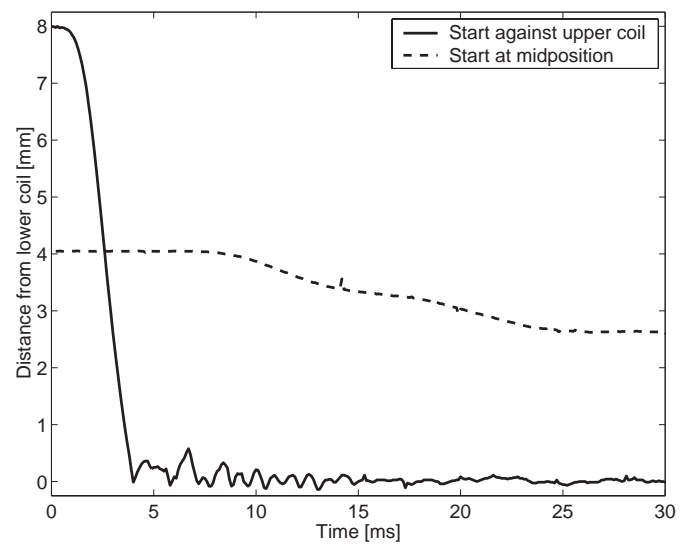

Fig. 8. Results of applying open-loop voltage for an equilibrium point $2.6 \mathrm{~mm}$ away from the lower coil.

the solution to an LQR optimization problem. In future work will we explore analytically the connection between performance and the selection of the control Lyapunov function to achieve insights into how to "tune" Sontag's feedback for performance. In addition the controller will be augmented with an integrator to eliminate the steady state tracking errors seen in Figs. 2 and 3 of Sec. 5.

\begin{tabular}{cccc}
\hline Parameter & Numerical value & Parameter & Numerical value \\
\hline$m$ & 0.27 & $k_{a}$ & 29.92 \\
$k_{s}$ & 158 & $k_{b}$ & 0.039 \\
$l$ & 4.0 & $k_{c}$ & 0.0012 \\
$b$ & 7.53 & $g_{1}$ & 3560 \\
$r$ & 6.0 & $g_{2}$ & 4820 \\
$\lambda_{\max }$ & 229 & $\alpha$ & 500 \\
\hline
\end{tabular}

Table 1. Numerical values of constants.

\begin{tabular}{cc}
\hline Numerical value of Q & For \\
\hline $\operatorname{diag}[1,4.7 e 4,10]$ & $z_{e q}=1.0$ and $z_{e q}=7.0$ \\
$\operatorname{diag}[1,6.6 e 4,10]$ & $z_{e q}=2.0$ and $z_{e q}=6.0$ \\
$\operatorname{diag}[1,1.2 e 5,10]$ & $z_{e q}=3.0$ and $z_{e q}=5.0$ \\
$\operatorname{diag}[1,5.3 e 5,10]$ & $z_{e q}=4.0$ \\
\hline Table 2. Numerical value of the matrix Q.
\end{tabular}

\section{REFERENCES}

Charara, A., J. DeMiras and B. Caron (1996). Nonlinear control of a magnetic levitation system without premagnetization. IEEE Transactions on Control System Technology 4(5), 513-523.

Cho, D., Y. Kato and D. Spilman (1993). Sliding mode and classical control of magnetic levitation systems. IEEE Control Systems pp. 42-48.

Fabien, B. (1996). Observer-based feedback linearization control of an electromagnetic suspension. ASME Journal of Dynamic Systems, Measurement and Control 118, 615-619.

Gentili, L. and L. Marconi (2003). Robust nonlinear disturbance suppression of a magnetic levitation system. Automatica 39(4), 735-742.
Green, S. and K. Craig (1998). Robust, digital nonlinear control of magnetic-levitation systems. ASME Journal of Dynamic Systems, Measurement and Control 120(4), 488-495.

Levin, M. and M. Schechter (1996). Camless engine. SAE 960581.

Lindlau, J. and C. Knospe (2002). Feedback lineariztion of active magnetic bearing with voltage control. IEEE Transactions on Control Systems Technology.

Mao, L. and J. Li (1999). Feedback linearisation of magnetic bearing actuators for a uniform upper bound of force slew rate. IEE-ProceedingsElectric-Power-Applications.

Munaro, C., M. Filho, R. Borges, S. Munareto and W. DaCosta (2002). Modeling and observerbased nonlinear control of a magnetic levitation system. IEEE Conference on Control Applications - Proceedings 1, 162-167.

Peek, R. and H. Wagar (1951). Magnetic design of relays. The Bell System Technical Journal pp. 2339.

Peterson, K. and A. Stefanopoulou (2003). Rendering the electromechanical valve actuator globally asymptotically stable. IEEE Conference on Decision and Control.

Rundell, A., S. Drakunov and R. DeCarlo (1996). A sliding mode observer and controller for stabilization of rotational motion of a vertical shaft magnetic bearing. IEEE Transactions on Control Systems Technology 4(5), 598-608.

Sepulchre, R., M. Jankovic and P. Kokotovic (1997). Constructive Nonlinear Control. Springer.

Shibukawa, K., T. Tsubakiji and H. Kimura (1991). Robust stabilization of a magnetic levitation system. Proceedings of the 30th IEEE Conference on Decision and Control 3, 2368-2371.

Smith, R. and W. Weldon (1995). Nonlinear control of a rigid rotor magnetic bearing system: Modeling and simulation with full state feedback. IEEE Transactions on Magnetics.

Sontag, E. (1989). A universal construction of artstein's theorem on nonlinear stabilization. Systems and Control Letters 13(2), 117-123.

Tai, C. and T. Tsao (2002). Control of an electromechanical camless valve actuator. Proceedings American Control Conference.

Velasco-Villa, M., R. Castro-Linares and L. CoronaRamirez (2001). Modeling and passivity based control of a magnetic levitation system. IEEE Conference on Control Applications pp. 64-69.

Wang, Y. (2001). Camless Engine Valvetrain: Enabling Technology and Control Techniques. PhD thesis. University of California Santa Barbara.

Yi, J., K. Park, S. Kim, Y. Kwak, M. Abdelfatah and I. Busch-Vishniac (1995). Force control of magnetic levitation system using flux density measurement. Proceedings of the 34th IEEE Conference on Decision and Control 3, 2153-2158. 